\title{
Actores, territorios y conflictos en políticas administrativas de la pesca artesanal. El caso de la zona contigua en la Región de Los Lagos y Aysén, sur de Chille ${ }^{1}$ \\ CATALINA ÁLVAREZ BURGOS \\ > Programa ATLAS, Universidad de Los Lagos, Chile. catalinalavarez@gmail.com
}

CLAUDIO GAJARDO CORTÉS

> Programa ATLAS, Universidad de Los Lagos, Chile. clgajardocortes@gmail.com

FRANCISCO THER RÍOS

> Programa ATLAS, Universidad de Los Lagos, Chile. fther@ulagos.cl

Universidad de Valparaíso

Facultad de Arquitectura

Revista Márgenes

Espacio Arte Sociedad

Actores, territorios y conflictos en políticas administrativas de la pesca artesanal. El caso de la zona contigua

en la Región de Los Lagos y Aysén, sur de Chile

Diciembre 2013 Vol. 10 NN$^{\circ} 13$

Páginas 79 a 90

ISSN elec. 0719-4463

ISSN imp. 0718-4034

Recepción: Agosto 2013

Aceptación: Diciembre 2013

\section{RESUMEN}

Durante siglos han existido desplazamientos de habitantes entre la Isla Grande de Chiloé, sus alrededores y el Archipiélago de las Guaitecas. Posterior a la regionalización del país y luego de las actividades pesqueras, los trayectos de pescadores de la región de Los Lagos hacia Aysén debieron gestionarse bajo una figura política y administrativa. Ésta incluye una zona contigua donde los gobiernos regionales negocian compromisos y compensaciones y una mesa público privada que define, desde el punto de vista político, científico, empresarial y pesquero-artesanal, la extracción de recursos en la zona. El presente trabajo describe las relaciones y estrategias que se despliegan en torno a ésta figura y la mesa de trabajo, identificando para ello a los actores, sus contextos discursivos y las dimensiones histórico-culturales. La capacidad de agencia en las prácticas y discursos de los actores da cuenta de un vínculo entre planos sociopolíticos y socio territoriales que, constituyendo una verdadera interfaz social, cuestionan la identificación dicotómica entre el Estado y las sociedades así como la verticalidad predominante en la visión y elaboración de las políticas públicas permitiendo nuevas lecturas y reflexiones tendientes a elaborar alternativas bajo una mirada política actualizada.

PALABRAS CLAVE

sociedades litorales, actores y conflictos territoriales, antropología política, recursos marítimo-costeros, pesca artesanal

Actors, territories and disputes in administrative policies of artisanal fishing. The case of the adjoining in the Los Lagos Region and Aysén Region, South of Chile

ABSTRACT

For centuries, there have been movements of inhabitants between Isla Grande of Chiloé, its surroundings and the Guaitecas archipelago. After the regionalization of the country and after fishing activities, the paths of fishermen in the region of Los Lagos to Aysén had managed under a political and administrative figure. This includes an adjoining area where regional governments negotiated compromises and trade-offs and a table private audience that set, from the point of view of political, scientific, business and artisanal fisheries, the extraction of resources in the area. This paper describes relationships and strategies that are deployed around the figure and the table of work, identifying to do this the actors, their discursive contexts and cultural dimensions. The capacity of agency practices and discourses of the actors gives an account of a link between socio-political plans and territorial partner which, constituting a true social interface, questioning the dichotomous identification between the State and the companies as well as predominantly vertical in the vision and development of public policies allowing new readings and reflections aimed at developing alternatives under a political look updated. KEYWORDS

coastal societies, actors and territorial disputes, anthropology policy, coastal resources, artisanal fishing

Actores, territorios y conflictos en políticas administrativas de la pesca artesanal. El caso de la zona contigua en la Región de Los Lagos y Aysén, sur de Chile > Catalina Álvarez Burgos, Claudio Gajardo Cortés, Francisco Ther Ríos
Revista Márgenes N ${ }^{0} 13$ Vol. 10 > Diciembre $2013>79$ Facultad de Arquitectura > Universidad de Valparaíso 
RÉSUMÉ

Les déplacements des habitants entre la grande île de Chiloé, ses environs et l'archipel des Guaitecas, ont existé pendant des siècles. Après la régionalisation du pays et des activités de pêche, les trajectoires des pêcheurs dans la région de Los Lagos à Aysén ont dû se gérer sous une figure politique et administrative. II s'agit d'un co-voisinage où les gouvernements régionaux négocient des compromis et des compensations, et d'une table publique-privée qui définie, du point de vue politique, scientifique, des affaires et de la pêche artisanale, l'extraction des ressources dans la zone. Cet article décrit les relations et les stratégies qui sont déployées autour de cette figure et la table de négociation, en identifiant, les acteurs, leurs contextes discursifs et les dimensions culturelles. La capacité des pratiques et des discours des acteurs rend compte d'un lien entre les plans sociopolitiques et socio territoriale qui constituent une véritable interface sociale, en questionnant l'identification dichotomique entre l'État et les entreprises aussi bien que la verticalité dominante dans la vision et l'élaboration des politiques publiques en ouvrant des nouvelles lectures et réflexions qui vise à développer de nouvelles solutions sous une vision politique actualiser.

MOTS CLÉS

société littorale, acteurs et conflits territoriaux, anthropologie politique, ressource maritime côtière, perche artisanale

\section{INTRODUCCIÓN}

La crisis ambiental implícita en los actuales modelos económicos está asociada a la apertura desregulada de recursos naturales al mercado internacional (Schaper, 1995; Leff, 1996). En este contexto la debilidad en el manejo y administración de los mismos remite a la falta de administraciones articuladas y a la debilidad de las políticas públicas para integrar a los actores del sector económico en cuestión (Pamplona, 2000; Bárcena et al., 2012). En los ambientes marinos, las políticas se han elaborado ya sea por departamentos fragmentando la concepción de los espacios de uso (Andrade, 2008) o bien excluyendo a sus actores relevantes (Alcalá 1999, 2003; Baliero et al. 2006). Las concepciones estáticas y compartimentalizadas subyacentes a ellas frenan el avance hacia miradas integradoras y dificultan la distribución de responsabilidades compartidas en torno a la explotación de los recursos naturales (CEPAL/PNUMA, 2012). El presente trabajo aborda una política de administración de recursos marinos a través del estudio de sus actores y relaciones. El estudio de ésta figura, conformada para responder a dinámicas socio-territoriales, da cuenta de procesos sociopolíticos alternos y brinda elementos críticos para la comprensión de políticas públicas en el sector de la pesca artesanal.

En las últimas décadas, los usos de espacios marítimo-costeros del sur de Chile han presentado un creciente dinamismo tanto en sus aspectos socioculturales (Buschmann y Fortt, 2005; Saavedra, 2008, 2011; Guerra y Skewes, 2008; Gelcich et al., 2010; Andrade et al. 2008; Skewes et al., 2012; Cordero, 2011), como en la aplicación de cuerpos legales. Son ejemplos de lo anterior la Política Nacional de Usos del Borde Costero (1994), la Ley General de Pesca y Acuicultura (1990) y la Ley de Espacio Costero Marino de los Pueblos Originarios (2008) (De la Maza et al. 2012; Martínez, 2012). Éstas normativas son implementadas siguiendo una lógica arriba-abajo (topdown) que omite la integración de las continuidades y discontinuidades socio-históricas en los territorios (Bugueño, 2011). En este sentido, las discusiones y avances en relación a la pesca han abordado tematicas tales como la orientación a la sustentabilidad en los cuerpos legales (Duhart, 2004), los cambios en las políticas de gestión (Olsson, et al. 2004; Arthur, 2005; Berkes, et al. 2001), la visibilización de redes de múltiples actores (Marín y Berkes, 2010; Crona et al., 2009; Marín, et al.,
2012) y el avance hacia la integración y participación en las figuras de administración de recursos naturales (Olsson et al., 2004a; Berkes y Carlsson, 2004; Berkes, 2006; Cundill, 2010; Keene et al. 2002; Gelcich et al. 2008).

De acuerdo a lo anterior, el estudio de aspectos socioculturales y su vinculación al campo de las políticas públicas, implica considerar que la dimensión sociopolíica actúa transversalmente a lo ambiental, territorial y cultural y se expresa en el modo en que éstos son problematizados por los actores.

Los estudios sociopolíticos han girado su mirada desde los sistemas de poder en culturas aisladas a la exploración de el lugar de la política en las sociedades contemporáneas (Abelés, 1997). Más que como una propiedad dada, el poder se manifiesta como relaciones expresadas en el ejercicio práctico e históricamente situado de normativas consensuadas y estrategias pragmáticas entre actores, instituciones y sujetos (Foucault, 2001). En la pesca, los contextos de incertidumbres, sobreexplotación de recursos y pérdida de referentes tradicionales conforman estrategias de superviviencia coexistentes a la mirada clásica de la política como convivencia (Abelés, 2008). En este sentido, estudios aplicados han abordado de nueva cuenta las relaciones sociedad-Estado (Hevia, 2009), la construcción social de sistemas políticos (De la Maza, 2009), los procesos de gobernanza y participación (Montecinos, 2007), las interrelaciones entre actores (Long, 2007), las alianzas públicoprivadas (Devlin y Mogullansky, 2009) y la direccionalidad en la aplicación de políticas públicas (Barragán y Wanderley, 2009).

El presente trabajo aborda el caso de la zona contigua en las regiones de los Lagos y Aysén y la mesa de trabajo asociada a ella. Se presenta una mirada antropológica centrada en la construcción cotidiana de sistemas político-culturales (De la Maza, 2009, 2012), el enfoque hacia los actores (Long, 2007) y el abordaje de sus prácticas y discursos como manifestación y ejercicio de relaciones de poder (Foucault, 2001). A través de un estudio cualitativo con fuentes etnográficas y documentales se describen aspectos históricos mostrando el modo en que esta figura se origina en respuesta a desplazamientos interregionales de pescadores artesanales y conforma una mesa público privada para administrar sus recursos ma-
80 > Revista Márgenes N 13 Vol. 10 > Diciembre 2013: 79-90 Facultad de Arquitectura > Universidad de Valparaíso
$>$ ISSN electrónico 0719-4436 $>$ ISSN impreso 0718-4034 
rinos. Se identifican los contextos discursivos de actores científicos, empresariales, administrativos y pesquero-artesanales reconociendo sus juegos de intereses y su posicionamiento relacional asi como la valoracion la diversidad socioterritorial de la pesca artesanal dentro del campo social presente en este sector económico.

\section{MATERIALES Y MÉTODOS}

Los resultados contribuyeron a generar antecedentes socio-políticos en relación a las sociedades litorales de Quellón y Melinka. Vinculadas por los usos históricos en el espacio marítimo intermedio a las regiones de Los Lagos y Aysén para su estudio se abordó como objeto de estudio la política administrativa de la zona contigua en donde ambas sociedades se encuentran protagonizando una divergencia de intereses. La perspectiva teórico-metodológica se centra en un análisis interactivo de los discursos y prácticas en torno a esta figura (Hevia, 2009). Los discursos de los actores, los espacios de interacción y la implementación de la política constituyen las principales unidades de estudio. En concordancia, los procedimientos siguieron un diseño cualitativo no experimental guiado por una conceptualización previa del objeto y problema de estudio (Torres y Becerra, 2006). Las etapas generales fueron; 1. Revisión documental, 2. Identificación de actores principales, 3. Diseño de instrumentos de recolección de información, 4. Entrevistas y conversaciones con expertos, 5. Trabajo de campo, 6. Sistematización de información y contraste de datos primarios y secundarios, 7. Interpretación y resultados.

En la etapa de revisión de fuentes secundarias se buscó perfilar la institucionalidad y los aspectos legales de la actividad pesqueroartesanal. En consideración de que el principal recurso de disputas correspondía al Erizo (Loxechinus albus), se revisó la prensa escrita y los informes emitidos por la Comisión de Manejo de Pesquerías Bentónicas (COMPEB). Ésta exploración permitió identificar los hitos históricos en la conformación de la zona contigua, los actores referenciados y los temas tratados en la mesa público privada. Posterior a la identificación de los actores se diseñaron instrumentos de recolección de información para cada uno de ellos. En la etapa de entrevistas y conversaciones con expertos se buscó reconocer el rol o auto-reconocimiento en el problema así como el reconocimiento de otros actores. Debido a las diferentes disponibilidades para acceder a los encargados y representantes, en algunos casos se recogió información primaria y secundaria, mientras que en otros sólo fue posible recoger sólo un tipo de información.

En la actividad en terreno se realizaron trabajos de campo hacia las dos sociedades litorales implicadas en el conflicto. Además se realizó una participación como invitada a una de las jornadas de la mesa público privada de la zona contigua. Durante la visita a las sociedades litorales se realizaron entrevistas a actores relacionados directa e indirectamente al problema. Se buscó caracterizar a los actores comparando sus discursos a la información de prensa previamente recogida. A partir de los relatos de pescadores entrevistados se buscó identificar los cambios en el desarrollo de la figura administrativa. Para esto se indagó respecto a un antes, un después, el presente y las proyecciones futuras. Durante la participación en la mesa público-privada se realizó un procedimiento de observación etnográfico y se complementó con entrevistas a algunos dirigentes de la pesca artesanal presentes. La participación tuvo el objetivo de distinguir y caracterizar a los actores, observar las dinámicas relacionales entre ellos, principalmente sus estrategias, e identificar los temas tratados y el modo en que los actores los desarrollaron.
En la etapa de sistematización se elaboró una matriz de información contextual sobre políticas públicas de pesca artesanal. Las entradas fueron la vinculación entre órganos administrativos y disposiciones legislativas, el desarrollo histórico de la ley de pesquera y los modelos de desarrollo económicos. Para la zona contigua se trabajó una matriz de discursos para cada uno de los hitos de la figura. En las entradas se agregaron notas y relatos asociados a las estrategias y percepciones sobre el pasado, en el presente y las proyecciones. Finalmente, en la interpretación y presentación de resultados se retomaron las preguntas de investigación a la luz de los discursos registrados. Éstas fueron las siguientes: ¿Qué plantearon los datos acerca de la relación entre maximización productiva y desarrollo sustentable? ¿Qué respuesta expresa el desarrollo de esta política frente a la problemática del sobre-explotación de recursos marinos? ¿Cómo influyeron las relaciones entre los actores la implementación de la política? ¿De qué modo aparece representada la complejidad sociocultural de la pesca artesanal?

De este modo, los abordajes de la Antropología del Estado (De la Maza, 2009; Hevia, 2009), la Sociología del Desarrollo (Long, 2007) y la Antropología Política (Abelés, 1997) resultaron pertinentes con el registro y permitieron despejar y clarificar los puntos críticos que responden a las preguntas anteriores. Así a partir del concepto de interfaz se aglomeró la información para identificar oposiciones o complementariedades en las relaciones entre los actores y los contenidos de sus discursos. El concepto de Co-construcción del Estado permitió evidenciar el carácter de emergencia y constitución de roles y valoraciones desde los actores y entre ellos en relación a la pesca artesanal y la sustentabilidad de la misma. Finalmente, la noción de lo político como sistema cultural permitió situar la conformación de esta política pública en el marco de la crisis ambiental de modelos económicos para el caso del sector de la pesca artesanal en Chile.

\section{ÁREA DE ESTUDIO}

El área de estudio corresponde al espacio que va desde los $41^{\circ}$ 20' $\mathrm{S}^{2}$ en el sector de Chacao, norte de la Isla Grande de Chiloé, a los $46^{\circ} 56^{\prime}$ S en la Península de Taitao, sector sur de la región de Aysén. En el borde costero asociado se distinguen caletas de pescadores de mayor tamaño poblacional asociadas a puertos de desembarque tales como Calbuco, Dalcahue, Quellón, Puerto Cisnes y Melinka. Asentamientos rurales ubicados en sectores alejados de centros poblados tales como Curaco de Velez, Pumillahue y San Juan. Asentamientos intermedios con mayor equipamiento y diversificación de actividades tales como El Manzano, Chonchi y Pichicolo, y asentamientos asociados a pequeños pueblos costeros tales como Cucao, Achao, Apiao, Catruman, Llanchid, Repollal y Quicaví.

Esta amplia zona incluye una diversidad de sistemas estuarinos, fiordos, islas y canales (Pickard 1971; Silva et al., 1995, citados en Molinet, et al. 2007). Además, corresponde a una de las cinco eco-regiones del país, con características de rica biodiversidad especialmente por invertebrados marinos, peces, aves y mamíferos (Hucke-Gaete et al., 2010). Posee una significativa riqueza patrimonial, con registros arqueológicos y patrimonios indígenas y de tradicionales chilotas. En el ámbito socio productivo se destacan prácticas relacionadas con el campo y mar (Bravo, 2004) que expresan una ancestral lógica consuetudinaria de usos en el borde costero (Skewes, et al. 2012). 
Dentro de la zona contigua trabajan 1.673 buzos en 1.212 embarcaciones. En total hay 4.709 personas en actividades extractivas, 1.500 como intermediarios y transporte y 4.786 personas en actividades de procesamiento en industrias. Considerando además los empleos indirectos habría 16. 493 personas trabajando dentro de la zona (Molinet et al., 2007). Las principales especies extraídas son la almeja (Venus antiqua), culengue (Gari solida), erizo (Loxechinus albus), cholga (Aulacomya ater), chorito (Mytilus chilensis), luga roja, (Gigartina skottsbergii), luga negra (Sarcothalia crispata), jaiba marmola (Cancer edwardsii), y macha (Mesodesma donacium).

En el trabajo sobre las especies anteriores se realizan jornadas diarias de extracción donde los pescadores van a las áreas de pesca y luego entregan a intermediarios. Para las especies de almeja, culengue y macha son trayectos de dos horas de navegación donde mediante buce ${ }^{3}$ o recolección (macha) trabajan en la extracción por períodos de cuatro horas (Ibíd.). Para la extracción del erizo, los pescadores pasan la noche en embarcaciones o en ranchas transitorias (Barahona et al. 2003). Las embarcaciones pequeñas son remolcadas por lanchas de transporte a los bancos naturales y realizan faenas diarias pernoctando en las cercanías. Durante la jornada los buzos extraen los recursos y los van entregando a lanchas transportadoras que realizan viajes diarios a los puertos de desembarque (Molinet, et al. 2007).

Para el trabajo sobre las especies erizo, luga roja y luga negra existen lanchas extractoras. Éstas se encuentran equipadas con camarotes y cocina por lo cual permanecen por períodos superiores a un día cercanos a bancos naturales (Gutiérrez et al., 2006). Para ir colectando las algas se utilizan aparejos de enmalle que pueden alcanzar los 80 Kg. (Molinet, et al. 2007). En el caso de la jaiba se identifican tres tipos de prácticas; i. jornadas diarias usando buceo y trampas, ii. jornadas de más de un día en embarcaciones mayores y iii. entrega a embarcaciones transportadoras hacia puertos de desembarque (Olguín et al. 2005). Las trampas son estructuras formadas por un enrejado de acero de forma cónica cubierta por una malla (Diámetro aprox. 100 cm.). En su interior se ubican carnadas de salmón, jurel, pejerrey y choritos (Molinet et al., 2007). La especie entra por la parte superior y queda atrapada debido a que en la boca superior de la trampa hay un plástico que impide su agarre en la malla.

\section{RESULTADOS}

\section{Hitos de la Zona Contigua}

En el desarrollo de la zona contigua, el primer hito corresponde a las pautas tradicionales en los desplazamientos de los pescadores artesanales. Su conformación, sin embargo, remite a los desplazamientos de grupos indígenas entre el mar interior de Chiloé y las Islas Guaitecas, el archipiélago de los Chonos y más al sur. Se trata de distintos grupos indígenas que desde los Archipiélagos de los Chonos y Guaitecas e incluso desde más al sur viajaban por el mar interior hasta llegar a la parte norte de la Isla Grande de Chiloé (Hucke-Gaete et al, 2008; Aspillaga et al., 2006; Álvarez, 2002; Legoupil, 2005; Emperaire, 1963). Con ello se conforma un corredor marítimo prehispánico estableciendo un tránsito longitudinal en el espacio marino actualmente entre las regiones de Los Lagos y Aysén. La apropiación de este espacio luego siguió conformando costumbres de desplazamiento entre los pescadores hasta la actualidad.

Un segundo hito histórico lo constituyen los viajes que las misiones jesuitas realizaron por este corredor. Para Legoupil (2005), la existencia temprana de cazadores-recolectores marítimos en las cercanías de Quellón ligados con los Chonos del Sur, explica la ayuda eficaz de los indígenas de Chiloé a las expediciones marítimas jesuitas de los Padres Venegas y García, o de Juan Vicuña, en estas zonas alejadas, en el transcurso del siglo XVIII (página 55). En general, los viajes de embarcaciones realizados durante la colonia eran guiados por los "prácticos" que eran indígenas con conocimiento sobre la geografía, el clima y las rutas de navegación (Bahamonde, 2006). Considerando lo anterior, es probable que de allí derive una continuidad en las rutas que unen la Isla Grande de Chiloé a las Guaitecas y el Archipiélago de los Chonos, traspasadas por los indígenas a los Jesuitas y colonizadores. Los viajes de las misiones partían desde Dalcahue y las cercanías de Quellón con dirección a Melinka. Entre sus motivaciones estaba la de traer habitantes originarios a lugares más cercanos para continuar con el proceso de evangelización (Ther Ríos, 2011).

Posteriormente, y hasta entrar el siglo XX, se conforman poblados de pescadores artesanales en ambas regiones. La colonización de Aysén, da cuenta de un eje de poblamiento insular para las Islas Guaitecas e islas Huichas, cuya tradición es Huilliche y cuya tendencia productiva es bentónica. Además un eje de poblamiento costero desde Piti-Palena hasta Puerto Aysén, caracterizado por actividades de pesca demersal (Martinic 2005 citado en Molinet et al., 2008; Saavedra, 2011). En dicho proceso, se refuerza la existencia de sistemas marítimos, desde la isla de Chiloé hasta zonas australes (...) como una zona de tránsito entre pueblos vecinos (Hucke-Gaete et al. 2008: 22). Estos poblamientos y la conformación de caletas y pueblos de pescadores va conformando un "pensamiento marítimo" cuyo tiempo transcurre principalmente 'entre caletas' (Munizaga, 1988:66). Su importancia radica en que la apropiación del espacio marino se expresa en trayectos entre localidades terrestres e insulares generando interconexiones socio espaciales duraderas en el tiempo.

En la constitución de 1828 el país es dividido administrativamente en ocho provincias ${ }^{4}$ siendo Chiloé una de ellas. Posteriormente en 1834 la provincia de Chiloé se divide en diez departamentos. En 1927 se establecen los límites del Departamento de Castro del modo siguiente:

El departamento de Castro estará formado por la parte de los actuales departamentos de Castro y Quinchao, que queda comprendida en la Isla Grande de Chiloé y los archipiélagos adyacentes, incluyendo la isla Chauque, el archipiélago de Quinchao, las islas Desertores, la de Guafo, y la parte del archipiélago de los Chonos, que queda al Poniente del Canal Moraleda y al Norte de los canales King y Pérez ${ }^{5}$.

Así, luego de diversas fusiones y cambios, durante las modificaciones realizadas en 1959 correspondientes a la creación de los departamentos de Palena, Aysén, Coyhaique y Chile Chico, la provincia de Chiloé queda dividida en los departamentos de Ancud, Castro, Quinchao y Palena ${ }^{6}$ (Mártinic, 2005). Hasta estas últimas modificaciones el departamento de Castro aún incluye las Islas Guaitecas, quedando integrados los vínculos que hasta el momento habían sido creados con la Isla Grande de Chiloé. En efecto, tras la migración de chilotes dedicados a las labores de explotación de Ciprés (Pilgerodendron uviferum), recolección de orilla, caza de lobos marinos y pesca artesanal, unidos por lazos parentales, costumbres, saberes y comercialización, se consolida un carácter identitario asociado a la población de Chiloé en el Archipiélago de las Guaitecas (Núñez, 2010; Hidalgo, 2011; Marticorena, 2009; Navarro, 2008).
82 > Revista Márgenes № 13 Vol. 10 > Diciembre 2013: 79-90 Facultad de Arquitectura > Universidad de Valparaíso
$>$ ISSN electrónico 0719-4436
> ISSN impreso 0718-4034 
La actual división administrativa que establece las regiones de los Lagos y Aysén data de 1974. En ella, el sector de las Islas Guaite$\operatorname{cas}^{7}$ deja de pertenecer a la Región de Los Lagos (Isla de Chiloé, Departamento de Castro) pasando a la Región de Aysén. Esto implica una nueva distribución de recursos estatales y el énfasis por la colonización del territorio. Sin embargo, se hace evidente que este corte tajante desconoce los usos tradicionales del territorio marítimo-costero lo cual deriva en la conformación de una administración obstructiva a los lazos parentales y rutas de navegación y extracción previamente consolidados. Sin embargo, los pescadores artesanales de la Región de los Lagos siguieron realizando sus faenas de pesca en las zonas del sector de Guaitecas, ahora pertenecientes a la Región de Aysén. Estos trayectos se mantuvieron sin mayores problemas, aun cuando la Ley General de Pesca y Acuicultura de 1991, estableció la regionalización del registro de pescadores (RPA) lo cual significaba que los pescadores pertenecientes a la Región de los Lagos no pudieran realizar prácticas extractivas en la Región de Aysén.

Entre el período que va desde 1980 hasta el 2000, presiones sobre los pescadores de la Región de Aysén dieron lugar a la emergencia de un conflicto con los pescadores que realizaban actividades en su sector. Entre ellas la aparición de marea roja en la Región de Aysén (Muñoz, et al., 1992; Medina, 1997). La toxicidad que genera en especies bentónicas tales como Chorito (Mytilus chilensis) y Cholga (Aulacomya ater), generaron una decaída de las prácticas tradicionales extracción y secado la Cholga. Además, la marea roja también alcanzó la Isla de Chiloé, específicamente en Quellón y otros sectores (Labra, 2011). En respuesta a dichos cambios, los pescadores se concentran en la extracción del erizo (Marticorena, 2009). Lo anterior se ve agudizado por la creciente explotación del erizo que, iniciando su explotación en el período de 1930 a 1976 con 5.000 ton. alcanza las 60.000 ton. entre 1994 y el 2002 (IFOP, 2012).

\section{Hitos en la conformación histórica de} la Zona Contigua Aysén-Los Lagos

El año 2001 representantes sindicales de Caleta Melinka, con motivo de impedir el flujo extractivo de pescadores de la Región de Los Lagos, recurren a un recurso de amparo para limitar a esta acción abogando a que dichas prácticas implican pérdidas económicas y ambientales. En respuesta, se generará una compensación económica ${ }^{11}$ de común acuerdo. En ella se permitiría la extracción de pescadores de libre tránsito entre ambas regiones según lo establecido en el artículo $\mathrm{N}^{\circ} 50$ de la Ley 18,892. En suma, este suceso como demanda social, se convierte en el punto decisivo a partir del cual se crea una mesa público privada. El 2005 se crea un Plan de Manejo para el recurso Erizo en la Zona Contigua. Alli se definen las cuotas a extraer y se monitorea su cumplimiento (Figura 1). Además se establecen compensaciones a los pescadores del sector sur por la extracción que realizan pescadores del sector norte (Molinet et al., 2007). Hasta ahora, los pescadores de la región norte, especialmente de Chiloé, reclaman derechos históricos sobre el sector marítimo de la región sur (Saavedra, 2007). Mientras que éstos últimos abogan por cuidar el sector y especialmente el recurso erizo.

\section{Caracterización de actores en el contexto de la Zona Contigua}

Los actores que participan en la mesa de la Comisión de Manejo de Pesquerías Bentónicas (en adelante COMPEB) representan a ambas regiones, y corresponden a funcionarios públicos, políticos,

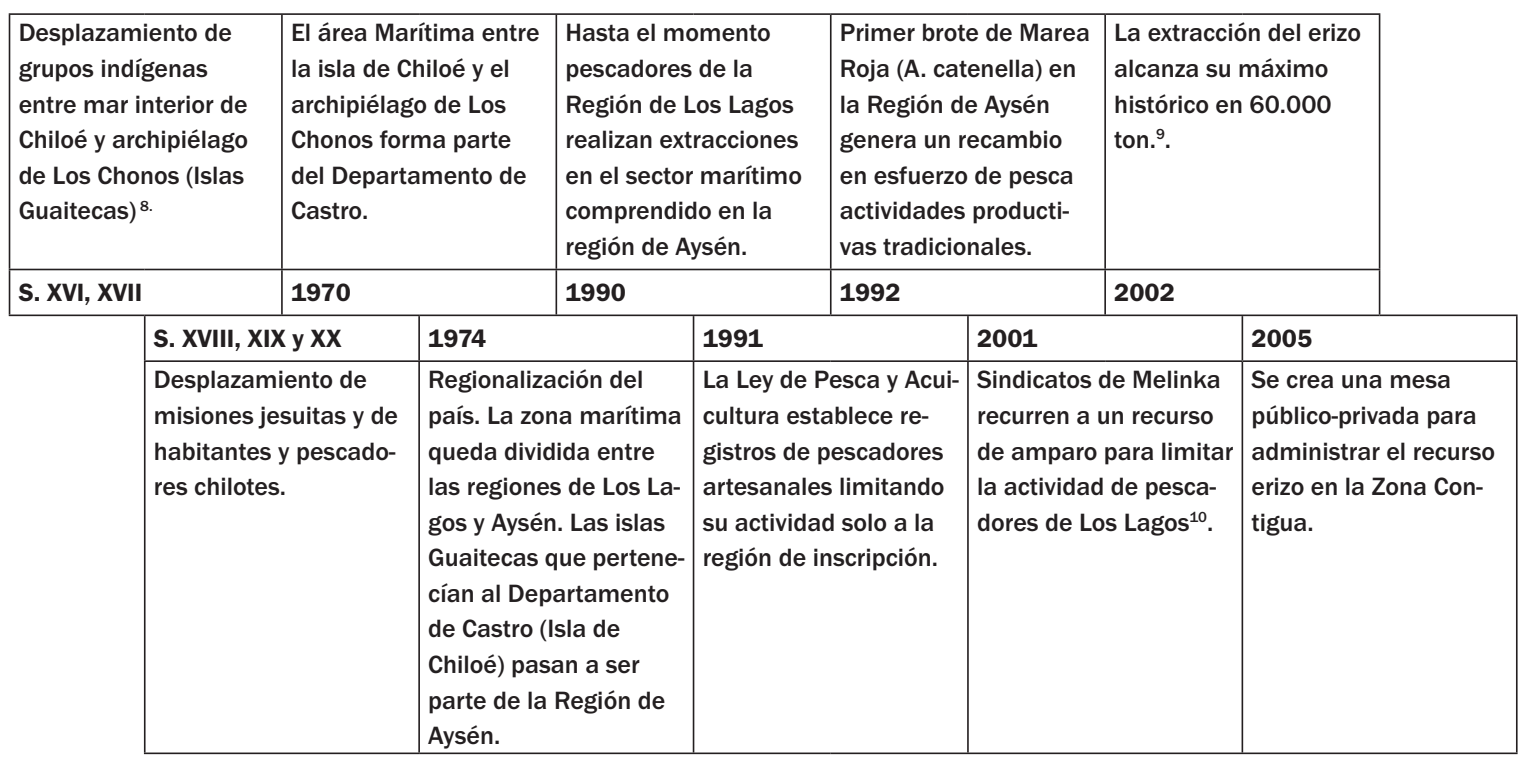

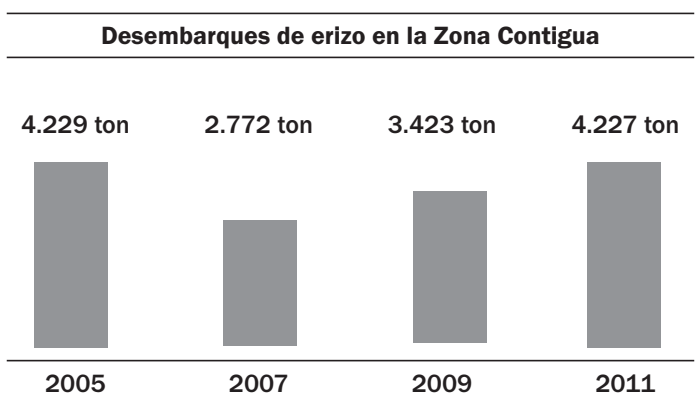

Actores, territorios y conflictos en políticas administrativas de la pesca artesanal. El caso de la zona contigua en la Región de Los Lagos y Aysén, sur de Chile > Catalina Álvarez Burgos, Claudio Gajardo Cortés, Francisco Ther Ríos
Figura 1. La figura muestra los momentos que dan cuenta de los
principales factores que contribuyeron a la conformación tanto del conflicto
entre los pescadores de Aysén y Los Lagos, como de la posterior conforma-
ción de la Zona Contigua y la mesa público privada (COMPEB). Las barras
bajo la figura muestran el inicio y la continuidad de la etapa científico-admi-
nistrativa del proceso. Éstas tienen la información sobre los desembarques
del recurso erizo administrado y delimitado por la mesa público privada para
esta especie

Revista Márgenes № 13 Vol. $10>$ Diciembre $2013>83$ Facultad de Arquitectura $>$ Universidad de Valparaíso 
representantes de pescadores artesanales, científicos, empresarios y funcionarios de orden público. Normalmente los actores son diferenciados como públicos o privados lo cual tiende a polarizar sus roles sociopolíticos. Evitando lo anterior se siguió un orden funcional a sus roles en la mesa público privada más por su relación al conflicto (Marin y Gelcich, 2012). En definitiva, se identificaron como macro actores territoriales, compuestos por otros diversos actores que pueden mantener relaciones contradictorias o conflictivas entre sí, a pesar de mantener factores de identificación común (P. ej. pescadores artesanales con diferencias territoriales).

Los funcionarios públicos, en tanto representantes del Estado se sustentan en una institucionalidad para desplegar sus roles. A partir de ahí establecen una racionalidad político administrativa en común a otros macro actores territoriales. Sin embargo, en su interior se distinguen funcionarios específicamente políticos de aquellos que se mantienen en una esfera técnica. Por otra parte, los pescadores artesanales, a pesar de tener un pasado común en sus prácticas de apropiación del litoral, se diferencian principalmente por el territorio. Su residencia en el sur de Chiloé o en el Archipiélago de las Guaitecas implica posicionamientos opuestos. En cuanto a los científicos, se distinguen los asociados a consultoras ${ }^{12}$ correspondientes en su mayoría a Biólogos Marinos e Ingenieros Acuícolas y los asociados al sector académico correspondientes a Ecólogos y Biólogos Marinos. En cuanto a los empresarios y a los representantes de orden público, en términos analíticos y funcionales, constituyen macro actores relativamente homogéneos. A continuación se presenta un extracto del diario de campo elaborado durante la visita a la mesa público-privada:

La mesa es itinerante, no tiene un lugar físico determinado para realizarse, y tampoco fechas estipuladas anualmente. Todo depende de las circunstancias en que se encuentre el mercado, la producción y el ambiente social. Reuniones pasadas se han realizado en Puerto Montt y Coyhaique. Melinka o Quellón se ven como lejanos para hacerlas por la falta de infraestructura o por ser lugares conflictivos y de posibles movilizaciones sociales (...) El espacio usado es alrededor de una mesa donde todos pueden verse las caras. Los temas de discusión son resolutivos. En la cabecera de la mesa se ubican las autoridades de gobierno de ambas regiones y los representantes institucionales del sector correspondientes a cada región. A un lado se encuentran los científicos y junto a ellos, también agrupados se encuentran los biólogos marinos que asesoran el plan de manejo. Por el otro lado se ubican los empresarios y los encargados del transporte y su comercialización. En el extremo se encuentran los representantes sindicales de los sectores de Quellón y Melinka ${ }^{13}$.

Considerando al territorio como construcción social, cambiante y en continuidad, siendo contenedor de múltiples relaciones a distintas escalas, redes de acción y acontecimientos, los procesos emergentes de estos actores dan cuenta de construcción de territorialidades en disputa por recursos, significados, control y legitimidad (Lefebvre, 2011; Ramírez, 2004; Long, 2007). De modo que la heterogeneidad de esta diferenciación se replica en las formas de construir, habitar y apropiarse de los territorios litorales.

\section{Relaciones entre actores}

Dentro del conflicto de la Zona Contigua, en el macro actor funcionario público, se distinguen los funcionarios políticos que tienden a ser conciliadores de intereses estatales, desarrollando estrate- gias normalizadoras de las actividades de otros actores, a través de sus constantes intervenciones con discursos que incorporan dimensiones del ser y deber ser definido en las normativas. Los funcionarios técnicos por su parte, aun cuando comulgan con los intereses estatales, experimentan cotidianamente las contradicciones de la aplicación de normativas por lo cual no se identifican sólo con su aplicación, sino que dan respuestas conciliadoras introduciendo adaptaciones a los contextos, situaciones y relaciones con los pescadores, sus dirigentes y territorios. En ambos casos, las acciones discursivas están dirigidas a no cuestionar los actuales ordenamientos, instituciones y sus funcionamientos, sino que a exigir que puedan ser observados y controlados desde el funcionamiento público hacia los actores del sector productivo. Ejemplo de ello es una recurrencia constante al control técnico de las actividades de pesca, tanto en la costa como en el mar abierto, con el fin de eliminar los desembarques ilegales, la extracción de recursos no autorizados y el mercado negro.

En términos socio territoriales el macro actor pescador artesanal posee un origen común, de manera que sus miembros comparten lazos parentales, costumbres, mitos y saberes tradicionales, producto de constantes migraciones entre Los Lagos y Aysén (Solari, et al. 2012). Se componen por un lado de los representantes de pescadores miembros de Caleta Quellón inscritos en el registro pesquero artesanal de la Región de Los Lagos. Su flota pesquera, de gran calado, es recurrentemente criticada por el otro grupo de pescadores aduciendo que dicha cualidad los excluye del sector propiamente artesanal. Por otro lado, integran a este macro actor los representantes de pescadores miembros de Caleta Melinka, pertenecientes a la comuna de Las Guaitecas e inscritos en el RPA de la Región de Aysén. Sus flotas son de menor calado en comparación a los pescadores de Los Lagos. Ellos tienen exclusividad de pesca en el polígono de exclusión ubicado alrededor de las Islas Guaitecas. Y además reciben aportes económicos para sus equipos, como compensación por la extracción de recursos por parte del otro grupo de pescadores en su región.

El macro actor científico se distingue en tanto se constituye de observadores de elementos de un ecosistema que sustentan procesos esenciales así como necesidades humanas, alejándose discursivamente de la visión asociada a recursos naturales, en tanto mercancías más o menos capaces de generar dinero (Berkes en Chirif, 2008). Sus discursos reflejan posturas de conservación (excluyendo al ser humano) o co-manejo (integración de instituciones y comunidades en torno a la administración de recursos naturales). Por tanto presentan un freno a concebir la biodiversidad marina sólo como recurso disponible para ser explotado. Se compone de científicos académicos asociados a instituciones universitarias que están en contacto con pescadores artesanales por medio de investigaciones o consultorías, En estos acercamientos han ido priorizando la investigación sobre biodiversidad marina más que los vínculos posibles entre ésta y las sociedades de pescadores artesanales. También componen a este macro actor las consultoras; organismos asesores, dispuestos por la ley para intervenir con el fin de apoyar el desarrollo y gestión económica de los pescadores artesanales. Ambos componentes sostienen discursos diferenciados sobre la disponibilidad de recursos marinos para su comercialización o su conservación. Su función es entregar los antecedentes necesarios que fijan las cuotas de extracción, a las cuales los pescadores pueden o no responder. Sus discursos reflejan ciertas relaciones conflictivas con los pescadores quienes, al no compartir
84 > Revista Márgenes N 13 Vol. 10 > Diciembre 2013: 79-90 Facultad de Arquitectura > Universidad de Valparaíso
> ISSN electrónico 0719-4436 > ISSN impreso 0718-4034 
sus prácticas extractivas y su relación con la biodiversidad, impiden avanzar en el mejor conocimiento del área.

El macro actor empresarios está compuesto por intermediarios y por la industria procesadora. Éstos hacen presente la dimensión de la cadena productiva de la pesca artesanal. En ella se exploran temas que van desde la compra en la playa hasta las empresas procesadoras y distribuidoras. No poseen conflictos entre sí y tienden a tensionar las relaciones al interior de los científicos y los pescadores artesanales. Ellos pueden ser determinantes en detonar el conflicto con las autoridades estatales. Al presionar por el constante aumento en la cuota de las especies rentables, introducen fuerzas contrarias a la aplicación de vedas abogando por requerimientos para su comercialización. Finalmente el macro actor del Orden Público, se compone de las Fuerzas Armadas. Es el actor más neutral. Su rol se limita fiscalizar normativas establecidas por la super vigilancia del Ministerio de Defensa Nacional que actúa por medio de la Subsecretaría para las Fuerzas Armadas. Esto lo hacen sin problematizar los usos de los espacios, sus actores o la disponibilidad y extracción de cuotas pesqueras. Son demandados por los pescadores artesanales para que ejerzan el cumplimiento de las normativas. Cuando las normativas tienen diversas interpretaciones, se observan ciertas presiones hacia las labores de este macro actor.

\section{Identificación de macro actores y sus discurso}

Esta primera caracterización de las relaciones consideró el contexto amplio del conflicto de la Zona Contigua. A continuación se pre- senta una segunda caracterización en relación a temáticas abordadas durante la mesa público privada. En esa dirección, más allá de encuadrar relaciones en subcategorías se pondrá el interés en las estrategias de conflicto y negociación entre los actores en busca de su potencial para encaminar posibles gestiones en la extracción de recursos o usos del borde costero.

Entre los temas tratados en la mesa de trabajo se distinguen unos más conflictivos que otros, tanto en un plano público como en el privado. Los problemas se construyen como escenarios que muestran a los macro actores territoriales actuando en bloque o por separado, incluso formando nuevas alianzas. Los temas principales son los modos de transporte de los recursos extraídos, los puntos de desembarque, la fiscalización de la marea roja, la evaluación en la disponibilidad de recursos y las condiciones sanitarias de los recursos extraídos.

Una de las estrategias desarrolladas por el macro actor Estado es elaborar mapas sociales y sobre ellos categorizar a los representantes de pescadores artesanales y funcionarios administrativos. Con ello se orienta a normalizar la actividad pesquero artesanal por medio del control y el conocimiento. En su consecución aparecen las funciones de los políticos administrativos quienes estandarizan la actividad manteniendo contacto con dirigentes y representantes políticos. Por otra parte, los funcionarios técnicos dirigen sus esfuerzos a realizar este cometido mediante alianzas con otros actores en trabajo en terreno.

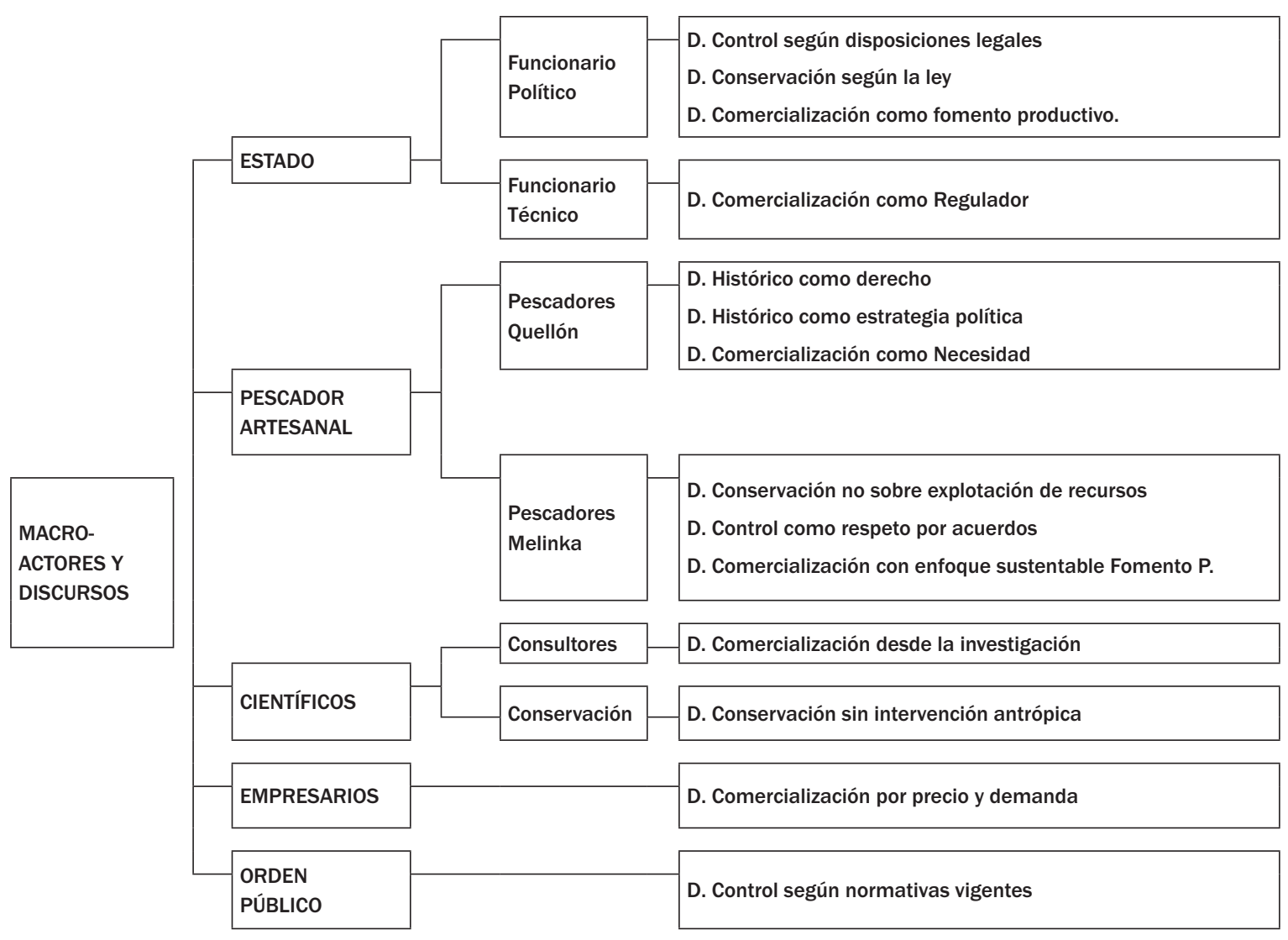

$>$ Figura 2. Los Macro Actores, compuestos por una diversidad, no poseen un discurso unitario, están vinculados a las instancias en que los necesitan. Son parte de las estrategias que desarrollan en relación con otros actores, y por tanto, los discursos no tienen los mismos sentidos para la totalidad de actores, cada uno de ellos los simboliza de manera distinta. Esto permite comprender lo que parece contradictorio, como que un actor esté hablando en términos de comercialización y sustentabilidad

Actores, territorios y conflictos en políticas administrativas de la pesca artesanal. El caso de la zona contigua en la Región de Los Lagos y Aysén, sur de Chile > Catalina Álvarez Burgos, Claudio Gajardo Cortés, Francisco Ther Ríos
Revista Márgenes № 13 Vol. 10 > Diciembre $2013>85$ Facultad de Arquitectura $>$ Universidad de Valparaíso 
El macro actor pescador artesanal con el tiempo ha logrado tecnificar su lenguaje hacia marcos de conocimiento biológicos, políticos y administrativos. Su tendencia es a desarrollar relaciones estratégicas con los funcionarios públicos por sobre su interés con los científicos. Sin embargo, los pescadores de Caleta Quellón trabajan constantemente con Biólogos Marinos de consultoras, con quienes presionan para mantener los niveles de extracción de sus flotas. Por su parte los pescadores de Caleta Melinka tienden a buscar asesoramiento jurídico tanto para regular el acceso de otras flotas; hacia Aysén hacen una apropiación del conocimiento científico sobre la conservación de su biodiversidad marina. Ellos trabajan mediante coordinaciones locales y, en contraste con los pescadores de Quellón, mantienen cierta distancia el macro actor Científico. En términos generales tienden a establecer relaciones estratégicas para poder participar en el sistema de mercado o invocando un enfoque conservacionista. Éste macro actor perfila el objetivo último consistente en negociar adaptando y conservando sus características como pescadores artesanales a las condiciones adversas a fin de mantener la actividad y el oficio.

El macro actor científico cumple un rol general de asesoramiento a la gestión de los pescadores. A pesar de que dichos vínculos se predispongan en los cuerpos de ley, ellos asumen que las relaciones y alianzas toman tiempo antes de consolidarse y por tanto actúan en función de aquello. Los Biólogos Marinos asociados a consultoras y los Ecólogos asociados a la academia poseen nociones y discursos conflictivos entre sí. Contrastan sus discursos respecto a la disponibilidad de los recursos; mientras los primeros abogan por una amplia disponibilidad de recursos en relación a las flotas pesqueras, los segundos argumentan que la disponibilidad de recursos se supone siempre como algo limitado.

\section{Discusión}

Desde el punto de vista de su desarrollo y sus actores, la imagen dinámica de esta política administrativa expresa ilustrativamente que la noción de un Estado jerárquico y centralizado puede ser desplazada por la noción de co-construcción del Estado. En efecto, los discursos desarrollados por los actores dan cuenta de una narración entre políticas de desarrollo, formas administrativas, legislaciones, funcionarios públicos, pescadores artesanales, empresarios y científicos. (Gómez, 2003; De la Maza, 2012; Schavelzon, 2008). Como política administrativa, la Zona Contigua manifiesta distintos significados y usos. En primer lugar los significados centrados en la interacción y representación desde donde es vista como un diálogo entre actores ${ }^{14} \mathrm{o}$ una instancia democrática participativa. En segundo lugar aquellos asociados a los conflictos desde donde se le entiende como una respuesta política frente a las demandas de los pescadores por la fijación de cuotas o bien como una estrategia que facilita el desarrollo de intereses económicos por sobre los ambientales. $Y$ en tercer lugar desde el ejercicio del control y la normalización, desde donde se ve como una vía para formalizar las prácticas pesquero-artesanales y a su vez generar formas de control frente a posibles movilizaciones sociales.

Las relaciones entre los actores y sus formas de apropiación de factores se contextualizan en la incertidumbre implícita a las instancias donde se logra la consecución de intereses. En general, cada actor, evidencia dinámicas relacionales en las cuales el modelo de desarrollo de la pesca artesanal muestra su cambiante conformación. Así por ejemplo, si bien el mercado muestra un aspecto determinante, también recibe determinaciones externas tanto en las restricciones de acceso a pesquerías como en las cuotas de extracción recursos, contexto en el cual los científicos muestran una dimensión activa en la resolución y producción de relaciones entre pescadores, mercado y estado (Díaz, 2008). En este contexto, más que sus asociaciones transitorias, la pesca artesanal adquiere un posicionamiento tendiente a dar continuidad tanto a su oficio como a sus formas de trabajo.

Las transformaciones histórico-regionales vinculadas al conflicto entre pescadores artesanales permitieron dar cuenta de ciertas formas de instanciación del oficio pesquero artesanal. Por medio de sus discursos los pescadores remiten a criterios constitutivos que recuperan a objeto de entrar en la disputa social ${ }^{15}$. Si bien tanto los pescadores de Quellón como los de Melinka son representados bajo la figura de pescadores artesanales ante la ley, las disparidades entre sus discursos expresan la flexibilidad de dicha categoría. Su puesta en duda desde unos pescadores a otros, expresa la apropiación y el uso bajo ciertos intereses y los mecanismos internos que surgen frente a condiciones que ponen en juego la continuidad del mismo. Aun con un origen territorial común, los pescadores de Melinka evocan criterios excluyentes asociados a la capacidad extractiva y el daño medioambiental que puede generar. En este sentido, las prácticas-pesquero artesanales y su vínculo con la biodiversidad marina se territorializa mediante conflictos internos, claramente derivados de la apertura económica del país.

Resalta en este escenario, el dinamismo y flexibilidad con que se manifiestan los contextos circunstanciales en que se desarrolla la problemática. Los discursos de los actores sobre un mismo aspecto normativo se encuentran fuertemente motivados por los intereses. A partir de lo anterior las interpretaciones divergen y la validez de las argumentaciones queda en segundo plano dando paso al uso y apropiación de conocimientos para sustentar estrategias de acción ya sea en busca de permanencia en ciertas áreas o de la emergencia de nuevas disputas dentro del conflicto. Este conjunto de significaciones dan cuenta de una Zona Contigua o arena política dinámica que, en efecto, ha sido reconocida por sus miembros como una alternativa para la participación y el diálogo. La imagen evidenciada en sus relatos y relaciones da cuenta de aspectos relacionales más que de imposiciones definitivas, concepción que permite ir más allá de la visión tradicional de verticalidad en la elaboración de políticas públicas (Long, 2007).

Considerando lo anterior, el uso de la etnografía es útil para la construcción de verosímiles (Maidana, 2013) que describen campos relacionales a partir de la observación de juegos de intereses. El carácter constructivo y cotidiano de las relaciones sociopolíticas permite avanzar desde una figura de administración a ciertas reflexiones sobre la pesca artesanal y sus relaciones con los actores del sector económico. A su vez, el enfoque centrado en el actor complementado con estrategias de triangulación de fuentes permite evidenciar la polifonía en torno a políticas públicas y de administración. El concepto de interfaz evidencia implícitamente la capacidad de agencia de los actores territoriales. Como estrategia de investigación permite concebir futuribles sobre lo que pueden generar dichas relaciones incorporando la incertidumbre a las respuestas que desarrollen. Se trata de un avance hacia una gestión flexible y ajustable a fenómenos coyunturales y con actores territoriales cambiantes. 


\section{CONCLUSIONES}

La zona contigua surge en estrecho vínculo a factores históricos y culturales presentes en las prácticas de pescadores artesanales de las regiones de los Lagos y Aysén. En este sentido constituye una instancia de conformación diferente al normal surgimiento de una política de administración. Dicho carácter permite evidenciar el campo relacional de la pesca artesanal en términos de las dinámicas internas a la figura de administración.

En la Antropología Política y su relación con estudios sobre la pesca artesanal, el enfoque centrado en el actor permite abordar la diversidad y complejidad de instancias contemporáneas de participación y juego de intereses en la sociedad. Bajo esta mirada los actores son reconocidos como agentes con la capacidad de apropiarse, transformar, negociar y resistir la influencia de factores exógenos y endógenos.

En contraposición a concepciones tales como el carácter depredador del pescador artesanal, esta perspectiva muestra el modo en que un actor elabora estrategias y utiliza conocimientos. En este sentido, la visión de la Zona Contigua como arena política deja abierta la puerta a reflexiones para una futura descentralización territorial y administrativa que apunte a una posible gestión de recursos y territorios, dando paso formas alternativas de participación ciudadana con reconocimiento del poder de actores sociales locales.

\section{BIBLIOGRAFÍA}

ABELÈS M. (1997) El lugar de la política. Revista Internacional de Ciencias. Sociales $\mathrm{N}^{\circ} 153$.

(1997) La antropología política: nuevos objetivos, nuevos objetos. Revista Internacional de Ciencias Sociales, páginas 51-72.

(2008) Política de la supervivencia. Buenos Aires: Eudeba.

ABRAMS P. (1988) Notes on the difficulty of studying the state. Journal of Historical Sociology, páginas 58-89.

ALCALÁ G. (1999) Con el Agua hasta los Aparejos: Pescadores y Pesquerías en el Soconusco. Centro de Investigaciones y Estudios Superiores en Antropología Social, Chiapas. 2003.

(2003) Políticas pesqueras en México (1946 - 2000). Contradicciones y aciertos en la planificación de la pesca nacional. México: COLMEX-CICESE-COLMICH.

(2003) Políticas Pesqueras en México: 1946-2000. Contradicciones y Aciertos en la Planificación de la Pesca Nacional. Colegio de México. Centro de Investigación Científica y de Educación Superior de Ensenada: El Colegio de Michoacán, México.

(2012) Pescadores en América Latina y el Caribe. Espacio, Población, Producción y Política (Volúmenes I y II). México.

ÁLVAREZ C. (2011) La política pesquero artesanal en Chile desde una aproximación antropológica: la Mesa Público Privada en la Zona Contigua, Regiones de Los Lagos y Aysén. Valdivia: Título para optar al grado de Licenciada en Antropología, Antropóloga. Escuela de Antropología. Facultad de Filosofía y Humanidades. Universidad Austral de Chile.

ÁLVAREZ R. (2002) Reflexiones en torno a las identidades de las poblaciones canoeras situadas entre los $44^{\circ}$ y $48^{\circ}$ de latitud sur, denominadas Chonos. Anales del Instituto de la Patagonia, serie Ciencias Humanas, (30):79-86.

ANDRADE B., ARENAS F. y GUIJÓN R. (2008) Revisión crítica del marco institucional y legal chileno de ordenamiento territorial: el caso de la zona costera. Revista de Geografía Norte Grande 41:23-48.

ARTHUR R. I. (2005) Developing, implementing and evaluating policies to support fisheries comanagement. MRAG Ltd, London.

ASPILLAGA E., CASTRO M., RODRÍGUEZ M., OCAMPO C. (2006) Paleopatología y estilo de vida: el ejemplo de los Chonos. Revista Magallania. (34):77-85.

BAHAMONDE J. (2006) Funciones en el Diario de Viaje i Navegación del padre García. Revista Estudios Filológicos. (41):19-30.

BALIERO W., BIASCO E., CONDE D., CORTAZZO R., FOSSATI M., GORFINKIEL D., LORENZO E., MENAFRA R., PÍRIZ C., y ROCHE I. (2006) Estudio de Base sobre el Estado del Manejo Costero Integrado en Uruguay: práctica, capacitación e investigación. Proyecto Sustentabilidad de la Zona Costera Uruguaya (AUCCCIDA). Universidad de la República, Montevideo/Dalhousie University.

BARAHONA N., ORENSANZ J. M., PARMA A. M., JEREZ G., ROMERO C., MIRANDA H., ZULETA A., CATASTI V., GÁLVEZ P. (2003). Bases Biológicas para rotación de áreas en el recurso erizo. IFOP, FIP 2000-18. Valparaíso.

BÁRCENA A., PRADO A., SAMANIEGO J. y PÉREZ R. (2012) La sostenibilidad del Desarrollo. A 20 años de la cumbre para la tierra. Avances, brechas y lineamientos estratégicos para América Latina y el Caribe. Naciones Unidas/Río+20.

BARRAGÁN R. \& WANDERLEY F. (2009) Etnografías del Estado en América Latina. Íconos, Revista de Ciencias Sociales. Número 34, 21-25.

BERKES F. y CARLSSON L. (2004) Co-management: concepts and methodological implications. Journal of Enviromental Management, 75:65-76.

BERKES F. (2006) From Community-Based Resource Management to Complex Systems. Ecology and Society 11(1): 45.

BERKES F., MAHON R., MACCONNEY P., POLLNAC R. y POMEROY R. (2001) Managing smallscale fisheries: alternative directions and methods. International Development Research Centre, Canada.

BRAVO J. M. (2004) La cultura chilota y su expresión territorial en el contexto de la globalización de la economía. Memoria para optar al Título Profesional de Geógrafo, Universidad de Chile, Escuela de Geografía, Santiago.

BUGUEÑO Z. (2011) Gobernanza y Uso del Territorio en Localidades Litorales. Caso de estudio: San Juan y Tenaún, Comuna de Dalcahue, X Región de Los Lagos. Memoria para optar al título de Geógrafo. Universidad de Chile.

BUSCHMANN A. y FORTT A. (2005) Efectos ambientales de la acuicultura intensiva y alternativas para un desarrollo sustentable. Revista Ambiente y Desarrollo, 21:3, 58-64.
Actores, territorios y conflictos en políticas administrativas de la pesca artesanal. El caso de la zona contigua en la Región de Los Lagos y Aysén, sur de Chile > Catalina Álvarez Burgos, Claudio Gajardo Cortés, Francisco Ther Ríos
Revista Márgenes № 13 Vol. $10>$ Diciembre $2013>87$ Facultad de Arquitectura > Universidad de Valparaíso 
CEPAL/PNUMA (2002) La sostenibilidad del desarrollo en América Latina y el Caribe: Desafíos y oportunidades. Comisión Económica para América Latina y el Caribe/Oficina Regional para América Latina y el Caribe. Santiago de Chile.

CORRIGAN P. y SAYER D. (2007) El Gran Arco. La Formación del Estado inglés como revolución cultural. En M. L. Lagos \& P. Calla, Antropología del Estado: Dominación y prácticas contestatarias en América Latina (páginas 39-116). La Paz: Cuaderno de Futuro $\mathrm{N}^{\circ} 23$.

CRONA B. y BODIN Ö. (2009) Social Networks and Leadership in Natural Resources Governance: are the important and how can we study them? Stockholm Resilience Center, Stockholm niversity, Stockholm, Sweden. Conference at the $7^{\text {th }}$ International Science Conference on the Human Dimensions of Global Environmental Change. International Human Dimensions Programme Open Meeting. The Social Challenges of Global Change.

CRUZ S. F. (2009) Formación del Estado, sujetos sociales, sociedad civil y ciudadanía: puntos teóricos de partida para una investigación sobre movilizaciones sociales. Revista Electrónica de Sociología, 2. Facultad de Sociología. Universidad Veracruzana.

CUNDILL G. (2010) Monitoring Social Learning Processes in Adaptive Co-management: Three Case Studies from South Africa. Ecology and Society 15(3):28.

DAS V. y POOLE D. (2008) El estado y sus márgenes. Etnografías comparadas. Cuadernos de Antropología Social №27, 19-52.

DE LA MAZA F. (2009) Etnografía del gobierno local: contingencias políticas y prácticas sociales en dos comunas de la Región de la Araucanía y Los Ríos, Chile. Pontificia Universidad Católica de Chile. VIII Reunión de Antropología del Mercosur.

(2009) Etnografía del gobierno local: contingencias políticas y prácticas sociales en dos comunas de la Región de la Araucanía y los Ríos, Chile. Pontificia Universidad Católica de Chile. VIII Reunión de Antropología del Mercosur.

(2012) Etnografía de las prácticas sociales y negociaciones en la política indígena actual, Araucanía-Chile. Rev. Austral Cienc. Soc. (22):85-100.

DUHART H. (2004) ¿Constituye la regulación actual una herramienta eficaz para la explotación sustentable del recurso pesquero? Memoria de prueba para optar al grado de Licenciado en Ciencias Jurídicas y Sociales. Universidad Austral de Chile. Facultad de Ciencias Jurídicas y Sociales. Escuela de Derecho.

EMPERAIRE J. (1963) Los Nómades del mar. Universidad de Chile. Santiago de Chile.

FOUCAULT M. (2001) Dits et écrits I y II. Paris:Gallimard.

GAJARDO C. y THER Ríos F. (2011) Saberes y Prácticas Pesquero-Artesanales. Cotidianidades y Desarrollo en las Caletas de Guabún y Puñihuil. Isla Grande de Chiloé. Chungará. Revista de Antropología Chilena, 43(1): 589-605.

GARCÍA R. (2006) Crítica de la Teoría de la Cultura Polítca. Política y Cultura, páginas 133-155.
GELCICH S., EDWARDS-JONES E., KAISER M. y CASTILLA J. C. (2008) Comanagement Policy Can Reduce Resilience in Traditionally Managed. Marine Ecosystems. 9(6):951-966.

GUERRA D. y SKEWES J. C. (2008) ¿Vernacularización, hibridación, enajenación o patrimonialización? Disyuntivas locales en la construcción del paisaje. Revista de Ciencias Sociales, páginas 5-37.

GUTIÉRREZ G., MATAMALA M. y HENRÍQUEZ J. (2006) Bases para el plan de manejo pesquerías bentónicas zona contigua Región X y XI. Consultora Pupelede.

HEVIA F. (2009) Relaciones sociedad-Estado: análisis interactivo para una antropología del Estado. Espiral, Estudios sobre Estado y Sociedad Vol. XV N 45, páginas 43-70.

HIDALGO C. (2011) La práctica pesquero artesanal y su vínculo con la expansión de la economía de capitales: aproximación antropológica a la pesca artesanal en las caletas de Islas Huichas, Puerto Cisnes y Puerto Melinka, Región de Aysén del General Carlos Ibáñez del Campo. Temuco: Tesis para optar al grado de licenciado en Antropología. Departamento de Antropología. Facultad de Ciencias Sociales y Jurídicas. Universidad Católica de Temuco.

HUCKE-GAETE R., ÁLVAREZ R., RUIZ J., TORRES J.P. (2008) Investigación para el desarrollo del área marina costera protegida de Chiloé. Primer Informe de avance. Proyecto BIP N³0040215-0. Universidad Austral de Chile.

INSTITUTO DE FOMENTO PESQUERO (IFOP) (2012) Ficha Erizo (Loxechinus albus). Recurso Objetivo Áreas de Manejo Pesca Artesanal - Chile.

KEENE S., GIBRÁN Y. y STOTZ W. (2002) Competing Visions for Marine Tenure and Co-Management: Genesis of a Marine Management Area System in Chile. Coastal Management 30:85-99.

LABRA G. (2011) Distribución espacial de quistes de Alexandrium Catenella y Protoceratium Reticulatum en dos sectores del litoral Norte de la Región de Aysén, Archipiélago de Las Guaitecas y Estero Pitipalena. Tesis para optar al Título de Ingeniero en Acuicultura. Universidad Austral de Chile.

LEFF E. (1996) La capitalización de la naturaleza y las estrategias fatales de la sustentabilidad. Boletín de la Red Ambiental. 7(16):17-20.

LEGOUPIL D. (2005). Recolectores de moluscos tempranos en el sureste de la isla de Chiloé: Una primera mirada. Magallania, Vol. 33(1):51-61.

LONG N. (2007) Sociología del Desarrollo: una perspectiva centrada en el actor. México: CIESAS.

MARÍN A. y BERKES F. (2010) Network approach for understanding small-scale fisheries governance: The case of the Chilean coastal co-management system. Marine Policy. 34(5):851- 858.

MARÍN A., GELCICH S., CASTILLA J. y BERKES F. (2012) Exploring Social Capital in Chile's Coastal Benthic Comanagement System Using a Network Approach. Ecology and Society 17(1):13. 
MARÍN A. y GELCICH S. (2012) Gobernanza y capital social en el comanejo de recursos bentónicos en Chile: aportes de redes al estudio de la pesca artesanal de pequeña escala. CURSHO. Cultural-Hombre-Sociedad, páginas 131-153.

MARTICORENA L. (2009) A pura memoria: Conocimientos y significados de la naturaleza en las localidades de Melinka y Repollal, Litoral norte de la Región de Aysén. Valdivia: Tesis para optar al titulo de licenciada en Antropología, Antropóloga. Escuela de Antropología. Facultad de Filosofía y Humanidades. Universidad Austral de Chile.

MARTÍNEZ V. (2012) Uso del espacio marítimo de los pueblos originarios y de los recursos hidrobiológicos. Efectos jurídicos. Memoria para optar al grado de Licenciado en Ciencias Políticas Jurídicas y Sociales. Universidad Austral de Chile.

MARTINIC M. (2005) De la Trapananda al Aysén: una mirada reflexiva sobre el acontecer de la Región de Aysén desde la prehistoria hasta nuestros dias. Santiago: Pehuén Editores.

MEDINA M. (1997) Estudio comparativo del fitoplancton del seno Aysén, $\mathrm{XI}$ región, y variables oceanográficas asociadas en cruceros de verano y otoño (1992). Tesis, Carrera de Biología Marina. Facultad de Ciencias del Mar. Universidad de Valparaíso, página 63.

MOLINET C., BARAHONA N., MATAMALA M. y otros. (2007) Diagnóstico biológico-pesquero para recursos bentónicos de la zona contigua, X y XI Región. Informe Final. Proyecto FIP ${ }^{\circ}$ 2005- 51. Universidad Austral de Chile. Entidad ejecutora.

MONTECINOS E. ( 2007) Los límites de la modernización municipal para profundizar la participación ciudadana en Chile ¿Es la gobernanza de proximidad una alternativa? Gestión y Organización. Volumen XVI, Número 2, 319-351.

MUÑOZ P., AVARIA S., SIEVERS H., PRADO R. (1992) Presencia de Dinoflagelados tóxicos del género Dinophysis en el seno Aysén, Chile. Revista de Biología Marina, 27 (2):187-212.

NAVARRO M. (2008) Comunidades Humanas y Poblaciones de Grandes Ballenas: Una aproximación desde la Antropología al patrimonio natural y cultural de las localidades del Archipiélago de los Chonos, Región de Aysén, Chile. Valdivia: Tesis de Grado para optar al título de Antropólogo y al grado de Licenciado en Antropología. Universidad Austral de Chile.

NÚÑEZ N. (2010) La transformación del trabajo como práctica económico cultural en comunidades de pescadores artesanales: nuevos significados a partir de la introducción y expansión de la industria salmonera. El caso de la comuna de Guaitecas, Región de Aysén. Temuco: Tesis para optar al Grado de Licenciado en Antropología. Departamento de Antropología. Facultad de Ciencias Sociales y Jurídicas. Universidad Católica de Temuco.

OLGUÍN A. y BARAHONA N. (2007) Análisis de la pesquería de jaiba en los principales centros de desembarque a nivel regional. Seguimiento del Estado de Situación de las Principales Pesquerías Nacionales: Proyecto Investigación Situación Pesquerías Bentónicas, 2006. IFOP - Subpesca, Informe Técnico $\mathrm{N}^{\circ} 3$.
OLSSON P., FOLKE C. y BERKES F. (2004) Adaptive Co-Management for Building Resilience in Social-Ecological Systems. Environmental Management, 34 (1):75-90.

OLSSON P., FOLKE C. y HAHN T. (2004a) Social-ecological transformation for ecosystem management: the development of adaptive co-management of a wetland landscape in southern Sweden. Ecology and Society 9(4): 2.

PAMPLONA F. (2000) Sustentabilidad y políticas públicas. Gaceta Ecológica. 56:46-53.

RIESCO V. (2010) Limitaciones al desarrollo de actividades pesqueras extractivas y acuícolas, a partir de la adopción de medidas de Zonificación del Borde Costero y el establecimiento de Áreas Protegidas Marinas. Santiago de Chile: Actividad Formativa Equivalente a Tesis para obtener el grado de Magíster en Derecho Ambiental. Facultad de Derecho, Universidad de Chile.

SAAVEDRA G. (2008) Desarrollo Endógeno y Dinámicas de Significación Cultural en el Borde Costero del Sur Austral Chileno. Simposio Antropoloxía e Cooperación ao Desenvolvemento (I Simposio de ALGA, Asociación Luso-Galega de Antropoloxía Aplicada).

(2010) Tradición e innovación en las comunidades de pesca artesanal del sur de chile: hacia un enfoque reflexivo del desarrollo endógeno. AIBR. Revista de Antropología Iberoamericana, vol. 7 núm. 1, página. 33-63,

(2011) Desarrollo, subjetividad y transgresiones identitarias en las costas del sur-austral chileno. Sociedad\&Equidad, páginas 282-303.

(2011) Perspectivas culturales del desarrollo en las costas australes de Chile. Aproximación antropológica a las persistencias y transformaciones de las economías de pesca artesanal en el litoral de Aisén. Memoria para optar al grado de doctor. Facultad de Ciencias Políticas y Sociología. Departamento de Ciencia Política y de la Administración. Universidad Complutense de Madrid.

(2013) La pesca artesanal en las encrucijadas de la modernización. Usos, apropiaciones y conflictos en el borde costero del sur de Chile. Revista andaluza de antropología. Número 4: encrucijadas para las sociedades pesqueras del sur en el marco de la globalización, páginas 79-102.

SCHAPER M. (1995) Comercio internacional y medio ambiente. Una perspectiva económica En: EI Desarrollo Sostenible en la economía de América Latina, CECODES, CLADEI. Santa Fe de Bogotá.

SHARMA A. y GUPTA A. (2006) The anthropology of the state: a reader. New Haven, Blackwell Pub.

SKEWES J. C., ÁLVAREZ R. y NAVARRO M. (2012) Usos consuetudinarios, conflictos actuales y conservación en el borde costero de Chiloé insular. Magallania (Chile), 40 (1): 109-125.

SOLARI M. E., SKEWES J. C., NAVARRO M., PAILLACHEO F. (2012) Historia ambiental de los archipiélagos de la Trapananda (Patagonia septentrional, Chile): desafíos para la conservación de la ballena azul.
Actores, territorios y conflictos en políticas administrativas de la pesca artesanal. El caso de la zona contigua en la Región de Los Lagos y Aysén, sur de Chile > Catalina Álvarez Burgos, Claudio Gajardo Cortés, Francisco Ther Ríos
Revista Márgenes № 13 Vol. $10>$ Diciembre $2013>89$ Facultad de Arquitectura $>$ Universidad de Valparaíso 
SUBPESCA (2012) Cuota de captura recurso erizo (Loxechinus albus) Regiones X y XI. Informe Técnico (R. Pesq.) N²4-2012.

SUBSECRETARÍA DE PESCA (2011) Informe Técnico (R. Pesq.) N 071-2011. Disminución Talla Mínima Legal del Recurso Erizo (Loxechinus albus, Molina) Regiones X y XI.

THER RÍOS F. (2011) Configuraciones del tiempo en el mar interior de Chiloé y su relación con la apropiación de los territorios marítimos. Desenvolvimiento y medioambiente, 23:67-80.

TORRES A. y BECERRA A. (2006) La construcción del objeto y los referentes teóricos en la investigación social. En: Becerra, A. y Torres A. (comp.). La práctica investigativa en ciencias sociales. (páginas 15-29). DCS, Departamento de Ciencias Sociales. UPN, Universidad Pedagógica Nacional, Bogotá.

TROUILLOT M-R. (2001) La antropología del Estado en la era de la globalización. Current Anthropology.

VALLEJOS A. (2009) Estudio de Caso. La Concertación para el Desarrollo de la Pesca Artesanal en la Región de Los Lagos. Osorno: CEDER - Universidad de Los Lagos.

NOTAS

1 Proyecto MEL 81100002 Valoración Económico-Ambiental de las Actividades Pesqueras Artesanales. Estrategias de Diversificación Productiva en Sociedades Litorales del Sur de Chile. Concurso Nacional de Atracción de Capital Humano Avanzado del Extranjero, Modalidad de Estadías Largas (MEL)/CONICYT - Chile.

2 Desde el Seno de Reloncaví, en las riberas de Maullín.

3 Corresponde al buceo Hocka, donde el pescador se equipa con un traje de goma, aletas, cinturón con plomos, mascarilla y regulador de aire. El buzo recibe el aire a través de una manguera conectada a un compresor ubicado en la embarcación.

4 Coquimbo, Aconcagua, Santiago, Colchagua, Maule, Concepción, Valdivia y Chiloé.

5 El último decreto que establece los límites del departamento de Castro es el DFL 8582 de 1928. (Cursivas mías).

6 Ley № 13.375. Crea y fija los límites de los departamentos de Palena, Aysén, Coyhaique y Chile Chico y las de sus respectivas comunassubdelegaciones. Consultado el 21 de Diciembre de 2013.

7 Guaitecas en Chesungun (lengua Huilliche, variante del Mapudungun) significa Paso al Sur (Navarro, 2008).

8 Han existido desplazamientos del norte a sur, constituyendo un corredor marítimo de grupos indígenas (Hucke-Gaete et al. 2008; Martinic, 2005).

9 Fuente: Subpesca (2012). Cuota de captura recurso erizo (Loxechinus albus) Regiones X y XI, 2012. Informe Técnico (R. Pesq.) $\mathrm{N}^{\circ} 24-2012$.

10 Se trata de pescadores artesanales de Chiloé (Molinet et al. 2008; Saavedra, 2011).

11 La compensación económica, sólo es para los pescadores inscritos en el sindicato (el único a la fecha) de Caleta Melinka, lo que excluye a otras caletas y sindicatos de la región de Aysén, tema no exento de conflictos. La compensación es llevada a cabo mediante la inversión en equipos para el mejoramiento de la actividad.

90 > Revista Márgenes Nº 13 Vol. 10 > Diciembre 2013: 79-90 Facultad de Arquitectura > Universidad de Valparaíso
12 El Plan de Manejo de la Zona Contigua está sujeto a la asesoría de una consultora, que mediante evaluaciones permite evaluar el estado de disponibilidad de los recursos a extraer. Trabajan biólogos marinos fundamentalmente.

13 Álvarez C. (2010) Diario de campo de la visita a la Mesa PúblicoPrivada de la Zona Contigua. Invierno de 2010.

14 Es necesario cuidar el no caer en observaciones individuales, más aún cuando nos encontramos en situaciones conflictivas. Esta situación hace necesario triangular información tanto en lo observado, como por lo obtenido en entrevistas, informes técnicos o prensa.

15 Universidad Austral. (2011). Aldo Mascareño y Juan Carlos Skewes, debate en la UACh sobre Cultura [Video]. Disponible en: http://www.youtube.com/watch?v=t61W3hhmSh4 